Journal of Animal and Veterinary Advances 11 (17): 3234-3241, 2012

ISSN: $1680-5593$

(C) Medwell Journals, 2012

\title{
Immunogenicity of Recombinant Attenuated Salmonella Choleraesuis C500 Strain Co-Expressing $M$ and $N$ Protein of Porcine Epidemic Diarrhea Virus (PEDV)
}

\author{
${ }^{1,2}$ Yu-Peng Ren, ${ }^{1,4}$ Xiao-Yu Wang, ${ }^{1,3}$ Qi-Gui Yan, ${ }^{1}$ Di-Shi Chen, ${ }^{1}$ Yu Cheng, ${ }^{1}$ Li Wan, \\ ${ }^{1}$ Lei Ma, ${ }^{1}$ Qi Zhang, ${ }^{1}$ Lin Guo, ${ }^{1}$ Zhi-Yong Xie and ${ }^{1,4}$ Wan-Zhu Guo \\ ${ }^{1}$ College of Veterinary Medicine, Sichuan Agricultural University, \\ 625014 Ya'an, Sichuan, P.R. China \\ ${ }^{2}$ College of Life Science and Technology, Southwest University for Nationalities, \\ 610041 Cheng'du, China
}

${ }^{2}$ Key Laboratory of Animal Disease and Human Health of Sichuan Province, ${ }^{3}$ Animal Biotechnology Center, Sichuan Agricultural University, 625014 Ya'an, Sichuan, P.R. China

\begin{abstract}
This study examined the recombinant Attenuated Salmonella Choleraesuis C500 containing $M$ and $N$ gene of PEDV whether can induce a systemic immune responses in mice. After identified by enzyme analysis and nucleotide sequencing test, the constructed recombinant plasmid, designated as pIRES-M-N was transfected into Vero cells. The transient expression protein was detected by indirect Immunofluorescence Assay (IFA) and Western-blotting. Result showed that pIRES-M-N can be expressed correctly in Vero cells. Then, the recombinant plasmid electroporated into S. choleraesuis C500 and the recombinant bacteria was proved to be stabile in vitro. The immunogenicities of this recombinant attenuated salmonella constructs were firstly investigated in a mouse mode. Specific anti-PEDV IgG antibodies and IFN- $\gamma$, IL-4 of cytokine were detected. The result showed that the recombinant Attenuated Salmonella could induce higher persistence specific hummune responses in mouse by contrast with naked DNA group after 42 days $(p<0.05)$. It also, stimulated a balanced Th1/Th2 immune response. That provided the clinical basic data and theoretical basis for success of the attenuated salmonella vaccine development.
\end{abstract}

Key words: PEDV, membrane protein, nucleocapsid protein, attenuated salmonella, immune response, China

\section{INTRODUCTION}

Porcine Epidemic Diarrhea (PED) is induced by Porcine Epidemic Diarrhea Virus (PEDV) and characterized with acute watery diarrhea, dehydration, vomiting in young piglets and leads to death with a high mortality rate. It causes important economic losses to the pig producer. Two commercial vaccines currently used to prevent $\mathrm{PED}$ are attenuated vaccine and inactivated vaccine. One of the attenuated porcine epidemic diarrhea virus derived from serial passage in Vero cells by Chang, designated KPEDV-9 could be applied as vaccine for protecting suckling piglets against PEDV infection (Kweon et al., 1999). Other studies showed that protective immunity of inactive vaccine of PEDV is probable. The titer of virus neutralizing antibodies in the blood and colostrums was in the range of 1:80-1:320 when used an inactivated vaccines for immunization of pigs both of which suffer from relatively low immune protective effect and lack duration of immunity (Sergeev et al., 2010).
With the rapid development of molecular biological technology, it has become a hot point of the research to develop new type gene engineering vaccines, characterized by safety, modifiability and effectivity. The Spike protein (S protein), Membrane protein (M protein), Nucleocapsid protein (N protein) and the Envelope (E) protein are the main structural proteins of the virus (Sun et al., 2006). S protein played important roles in virus entry, neutralization and pathogenicity has the ability to induce specificities of neutralizing antibodies. But $S$ gene also has relatively evident variability (Sato et al., 2011; Kang et al., 2005); M protein directs the nucleocapsid into the budding particle (Chen et al., 2008) and may stimulate the body produce more $\alpha$-interferon (Jinghui and Yijing, 2005). Using PEDV modified whole $M$ protein genes as candidate genes, Zhang et al. (2011) constructed prokaryotic expression plasmid and reacted with the rabbit anti-PEDV serum indicated that $\mathrm{M}$ protein possessed reactiveness. The highly conservative gene of PEDV nucleocapsid protein gene

Corresponding Author: Qi-Gui Yan, College of Veterinary Medicine, Sichuan Agricultural University, 625014 Ya'an, Sichuan, P.R. China 
was demonstrated by PCR and bioinformatic analysis (Lee and Yeo, 2003). The recombinant Lactobacillus casei 393 expressing PEDV N protein constructed by Ge et al. (2009) can activate the cellmediated and antigen specific T-cell immune response. These studies proved that PEDV M,N gene may be used as an important candidate gene for the research of gene engineering vaccine.

Attenuated Salmonella allow the administration of recombinant vaccines via the mucosal surfaces. The bacteria used for DNA vaccine delivery either entered the host cell cytosol after phagocytosis by the APC (Antigen-Presenting Cells). They colonize the mucosa and other lymphoid tissues for prolonged periods of and induce mucosal and systemic immune responses both humoral and cell mediated (Sirard et al., 1999; Dietrich et al., 2009).

In order to develop a new kind of vaccine of PED using $P I R E S$ as target gene, $P E D V M, N$ as aim gene, researchers constructed a recombinant plasmid, designated as pIRES-M-N. Then, the recombinant attenuated salmonella choleraesuis C500 strain harboring the pIRES-M-N was constructed and immune tested in mice. Results showed that the recombinant attenuated salmonella choleraesuis C500 strain entered the epithelial lining of the gut to persistently provide the recombinant plasmid could enhance immune function of PIRES-M-N. It also provides theoretical data for clinical application fundamental data and establish foundation for further development of new vaccine of PED.

\section{MATERIALS AND METHODS}

Plasmid, strains and animal: PEDV-DY isolate $M, N$ protein gene full-length cDNA amplified by RT-PCR. Two pairs of primers for RT-PCR were designed and synthesized according to the sequence of PEDV CV777 strain (GenBank Accession No. AF353511). One primer pairs used were P1 (TCTAGAAGATG TCTAACGGTTCTATTCC) and P2 (GTCGACTAGAC TAAATGAAGCACTT) for he $M$ gene of PEDV, the Xba $\mathrm{I}$ and Sal I sites are underlined. One primer pairs used were P3 (CTCGAGATGGCTTCTGTCAGTTT TCAGGA TCG) and P4 (ACGCGTATTTCCTGTGTCGAAGATCT CGTTGA) for the $N$ gene of PEDV, Xho I and Mlu I sites are underlined. Then, $M$ and $N$ gene were cloned in PMD19-T, respectively designated as pMD19-T-M, pMD19-T-N. pMD19-T simple vector was production of TaKaRa Biotechnology (Dalian) Co., Ltd.; E. coli DH5 $\alpha$ was used as the host strain for construction of vectors.
Salmonella choleraesuis C500 and Eukaryotic expression vector pIRES kindly provided by Chen DS, a doctor at the Animal Biotechnology Center of Sichuan Agricultural University. PEDV inactivated vaccine was production of PULIKE Biological Engineering INC. About 4 weeks old $\mathrm{BALB} / \mathrm{c}$ mice were purchased from Laboratory Animal Research Center of SiChuan University.

Reagent and instrument: T4 DNA Ligase, various restriction endonuclease were purchased from TaKaRa Biotechnology, Dalian, China. Goat anti rabbit IgG-FITC was purchased from Zhong Shan-Golden Bridge Biological Technology, Beijing, China. Protein marker was purchased from TaKaRa Biotechnology, Dalian, P.R. China. FuGENE ${ }^{\circledR} \mathrm{HD}$ Transfection Reagent, Endo-Free Plasmid kit I were purchased from Omega Biotech, Shanghai, China. PEDV Antibody ELISA kit was purchased from Colorful Gene Biotech Co. Ltd. WuHan, China. IgA ELISA detection kit of Mouse and IFN- $\gamma / \mathrm{IL}-4$ ELISA detection kit of Mouse were purchased from Excell Biotech, Shanghai, China. Rabbit anti-PEDV serum are kept in the laboratory. Inverted fluorescence microscope was purchased from Nikon Instruments, Shanghai, China.

\section{Construction of pIRES-M and pIRES-N and pIRES-M-}

$\mathrm{N}$ : pIRES vector contains the cloning sites (MCS A and

B) an arrangement that allows cap-indpendent translation of the gene cloned into MCS B. The, $1321 \mathrm{bp}$ nucleotide sequence of $N$ gene was retrieved by double-enzyme digestion by Xho I/MluI and cloned into MCS A of pIRES vector that obtain the eukaryotic recombinant expression plasmids PIRES-N.

The $681 \mathrm{bp}$ nucleotide sequence of $M$ gene was retrieved by double-enzyme digestion by Xba I/Sal I. Then it was respectively cloned into MCS B of pIRES and of pIRES-N that obtain the eukaryotic recombinant expression plasmids pIRES-N and pIRES-M-N.

\section{The expression of recombinant plasmid in Vero cell Transfection: pIRES-M, pIRES-N and pIRES-M-N expression plasmid DNA were extracted using Endo- Free Plasmid kit I. Vero cells were grown to $70 \%$ in a six well plate and then transfected with the expression vectors using FuGENE ${ }^{\circledR} \mathrm{HD}$ transfection Reagent as specified by the manufacturer. At the same time, blank and PIRES were the control groups.}

Indirect Immunofluorescence Assay (IFA): Transfected cells were washed after $48 \mathrm{~h}$ of growth and fixed in 
acetone reacting with rabbit anti-PEDV serum and goat anti rabbit IgG-FITC. At the same time, the control group pIRES regarded as reference using the method above. The cells were observed and photographed under inverted fluorescence microscope.

Western-blotting analysis: The collected precipitation was washed twice with a Phosphate Buffer Solution (PBS) lysed in lysis buffer and boiled for $10 \mathrm{~min}$ at $72 \mathrm{~h}$ after transfection. The supernatant after centrifugation were assayed by Sodium Dodecy Sulfate-Polyacrylamide Gel Electrophoresis (SDS-PAGE) and Western-blotting with rabbit-anti $P E D V$ serum as primary antibody and goat anti-rabbit IgG marked with alkaline phosphatase as secondary antibody.

\section{Construction of the recombinant bacteria}

The recombinant bacteria constructed: Plasmid pIRES-M-N was electroporated as described previously (Schodel, 1990) into S. choleraesuis C500. The resultant recombinant strain is called SC. 500 (pIRES-M-N). At the same time, plasmid pIRES electroporated into $S$. choleraesuis C 500. The resultant recombinant strain is called SC. 500 (pIRES).

In vitro plasmid stability: The persistence of a plasmid in cells of S. choleraesuis C500 in vitro determined by the earlier methods (Dunstan et al., 2003). Bacterial cultures were serially passaged for approximately 100 generations ( 10 generation times of $12 \mathrm{~h}$ ) without ampicillin selection (Chen et al., 2005). the bacteria were sampled every $12 \mathrm{~h}$ then cultured in $\mathrm{DHL}$ agar overnight at $37^{\circ} \mathrm{C}$ without ampicillin or with either $75 \mu \mathrm{g} \mathrm{mL}{ }^{-1}$ ampicillin. The percentage stability of the plasmid was estimated by calculating the number of cells containing the plasmid at each passage (i.e., bacterial colony counts on DHL agar containing ampicillin) divided by the total number of cells at each passage (viable count on $\mathrm{DHL}$ agar).

Animal grouping and immunity: About 6 weeks old female BALB/c mice were assembled into 6 groups of 8 animals with each group having similar weight. The four of groups were then allocated to be immunized orally on days 0,14 and 28 , respectively with $1 \times 10^{8}$ CFU SSC. 500 (pIRES-M-N)/0.2 mL, $1 \times 10^{8}$ CFU SC. 500 (pIRES-M$\mathrm{N}) / 0.2 \mathrm{~mL}, 1 \times 10^{8}$ CFU SC. 500 (pIRES) $/ 0.2 \mathrm{~mL}$ and $0.2 \mathrm{~mL}$ of $8 \%$ sodium chloride then the other two groups were injected into the femoribus internus muscle, respectively with inactivated vaccine and $0.1 \mathrm{mg}$ of plasmid DNA contianing pIRES-M-N. Procaine hydrochloride was injected into the same position 3 days before immunization. After immunization, the mouse sera were obtained by tail tip blood sampling at different time points and stored at $-20^{\circ} \mathrm{C}$.
Bacterial colonization of organs in vivo and plasmid stability in vivo: One group of the SC. 500 (pIRES-M-N) orally immunized mice were used in this test.

Following removal, spleens, livers and feces were homogenised separately in sterile plastic bags, containing $5 \mathrm{~mL}$ of PBS using a glass homogeniser (Dunstan et al., 2003). The number of plasmid-bearing bacteria in each phase were quantified on LB agar containing ampicillin $\left(75 \mu \mathrm{g} \mathrm{mL}{ }^{-1}\right)$. The numbers of bacteria present in the spleens, livers and feces were determined by viable count.

ELISA for antigen-specific IgG antibodies: The PEDV-specific IgG antibodies Enzyme-Linked Immunosorbent Assay (ELISA) as described by the manufacturer's instructions of PEDV Antibody ELISA kit.

IL-4 and IFN- $\boldsymbol{\gamma}$ cytokine ELISA: The IL-4 and IFN- $\boldsymbol{\gamma}$ cytokine levels in sera were measured using PharMingen OptEIA ${ }^{\mathrm{TM}}$ ELISA kits according to the manufacturer's instructions.

\section{RESULTS}

Construction and identification of eukaryotic recombinant expression plasmid: Enzyme identification results confirmed that $\mathrm{pIRES}-\mathrm{M}$, pIRES-N and pIRES-M-N were constructed successfully (Fig. 1 and 2).

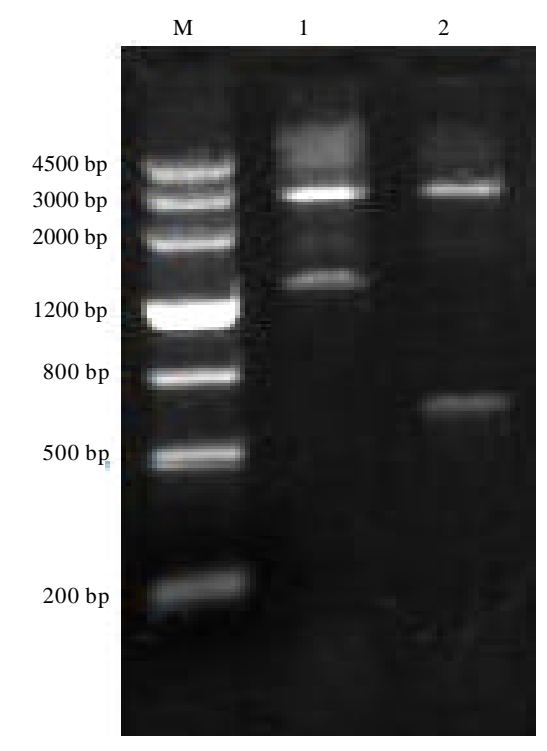

Fig. 1: Enzyme digestion profile of pIRES-N, pIRES-M. M: Marker; 1: Xho/Mlu I enzyme identification of plasmid pIRES-N (1329 bp); 2: Xba/Sal I enzyme identification of plasmid pIRES-M (691 bp) 


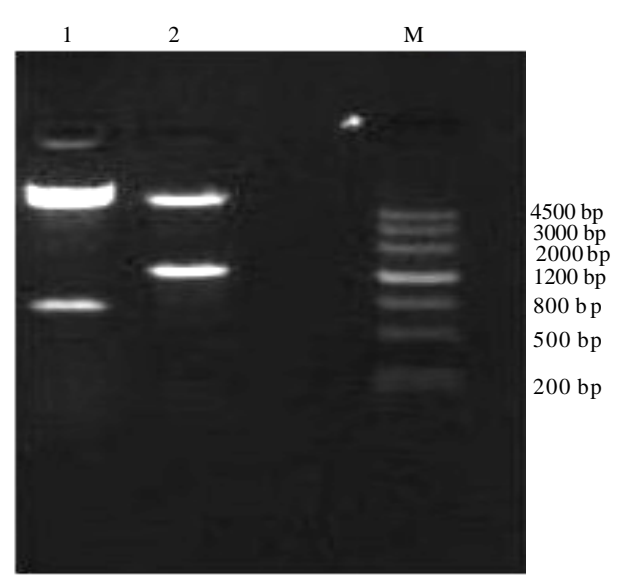

Fig. 2: Enzyme digestion profile of pIRES-M-N. M: Marker; 1: Xba/Sal I enzyme identification of plasmid pIRES-M-N (691 bp); 2: Xho/Mlu I enzyme identification of plasmid pIRES-M-N (1329 bp)
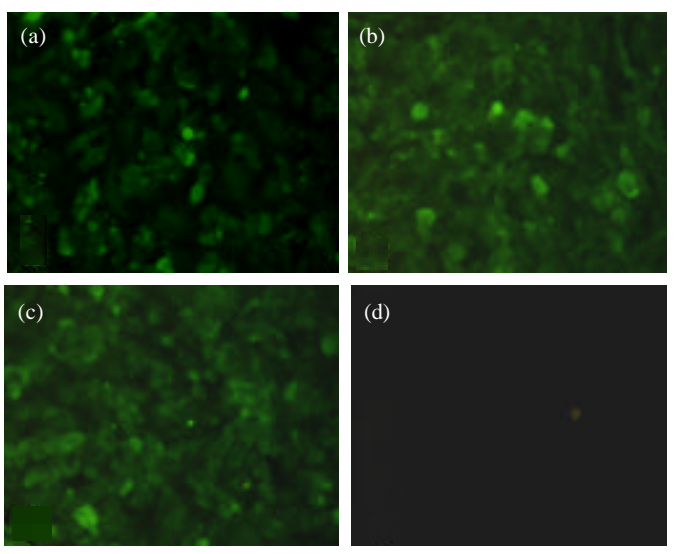

Fig. 3: IFA test of Vero cells transfected by recombinan plasmids DNA (400x). a) pIRES-M; b) pIRES-N; c) pIRES-M-N and d) pIRES

Expression of recombinant plasmid in Vero cells FA detectionl: Vero monolayer cells were transfected with pIRES-M, pIRES-N and pIRES-M-N and pipette onto the cell glass slide. Transfection was observed with inverted fluorescence microscope. Specific green fluorescence was observed in the cytoplasm of Vero cells transfected with pIRES-M, pIRES-N and pIRES-M-N but not in the control group which was transfected with pIRES (Fig. 3).

Western-blotting analysis: The cells transfected with pIRES-M, pIRES-N and pIRES-M-N were cracked and tested by SDS-PAGE. The blot was then reacted with rabbit-anti PEDV hyperimmune serum. In the immunoblot analyses, two reaction bands were observed at 24.2 and $48.8 \mathrm{kDa}$ and the sizes were consistent

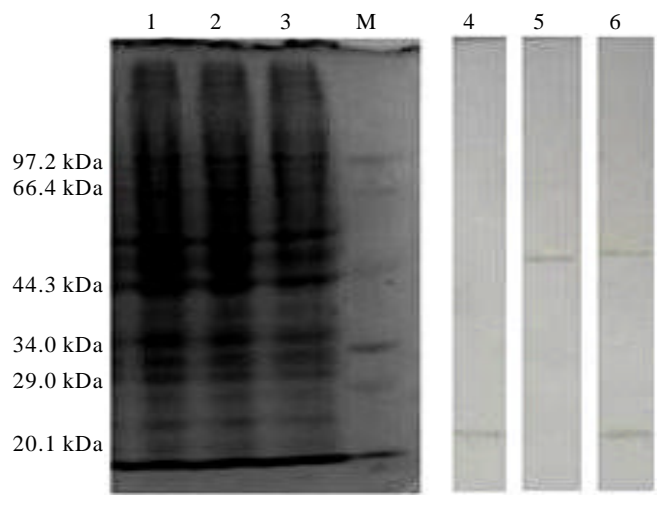

Fig. 4: Expression of $\mathrm{M}$ and $\mathrm{N}$ protein in Vero cells detected by Western-blotting. M: High molecular weight protein marker; 1: Express result of pIRES-N; 2: Express result of pIRES-M; 3: Express result of pIRES-M-N; 4: pIRES-N result of Western-blotting: 5; pIRES-M result of Westernblotting; 6: pIRES-M-N result of Western-blotting

with expectation which proved the successful expression of the constructed recombinant plasmid (Fig. 4)

In vitro plasmid of the recombinant bacteria stability: The maintenance of each plasmid during growth of Salmonella choleraesuis C500 in vitro was assessed in media with and without antibiotic selection to investigate the affect of an antibiotic-free environment on plasmid segregation. SC. 500 (pIRES-M-N) was grown without antibiotic selection $>120$ generations each point represents the percentage of the bacterial population that has retained the plasmid (Fig. 5).

No plasmid-containing bacteria were isolated from the SC. 500 (pIRES-M-N) culture after approximately 120 generations of growth in antibiotic-free media. Recombinant plasmid pIRES-M-N was stably maintained by SC. 500 relatively with $>70 \%$ of the population retaining the plasmid after 80 generations of growth in antibiotic-free media (Fig. 5).

\section{Animal grouping and immunity}

Bacterial colonization of organs and plasmid stability in vivo: One group of $\mathrm{BALB} / \mathrm{c}$ mice were immunized orally with SC. 500 (pIRES-M-N). The plasmid stability and the persistence of the bacteria in vivo was investigated $>15$ days. The $1,3,5,7,9,11,13$ and 15 days postimmunisation the spleens, livers and feces were removed from immunised mice. Plasmid stability was defined by the recovery of bacteria on media with ampicillin. Plasmidcontaining SC. 500 (pIRES-M-N) was isolated from the feces of SC. 500 (pIRES-M-N) immunised mice on day 1 


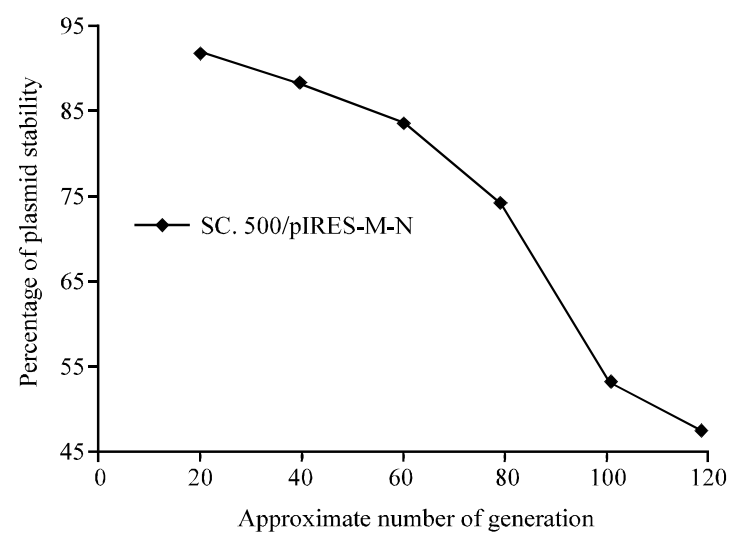

Fig. 5: In vitro stability of recombinant plasmid in SC. 500 (pIRES-M-N) was passaged for approximately 120 generations in antibiotic-free media. Overnight broths were subcultured and the percentage of bacteria containing the plasmid (antibiotic resistant) was determined by viable count on media with and without the relevant antibiotic)

only whereas immunised mouse had a small number of plasmid-containing bacteria isolated from the spleens and livers after 5 days. Number plasmid-containing SC. 500 (pIRES-M-N) was isolated from all of immunised mice after 2 weeks (Fig. 6).

ELISA for antigen-specific IgG antibodies: The mouse sera collected at different time points (the random sampling of five mice per group of eight were selected) after immunization were used to measure levels of specific anti-PEDV IgG. Anti-PEDV IgG level in mice immunized with inactived vaccine were greatly increased after 7 days $(\mathrm{p}<0.05)$. By contrast, the recombinant bacteria immunized group serum levels of specific anti-PEDV IgG did not elevate clearly. Anti-PEDV IgG level in mice immunized with naked DNA (pIRES-M-N) have shown an upward trend as the recombinant bacteria immunized group at 21 st day. The highest titer of the naked DNA group was observed 1 week after the third immunization. At this time, the recombinant bacteria group serum IgG level was close to the naked DNA group's $(\mathrm{p}>0.05)$.

After 42 days, the level of naked DNA group serum in mice has dropped but the recombinant bacteria immunized group has continued to rise (Fig. 7). By the experiment, the immunity effect of inactived vaccine was better than recombinant bacteria. However, it also showed that the recombinant bacteria could persistently

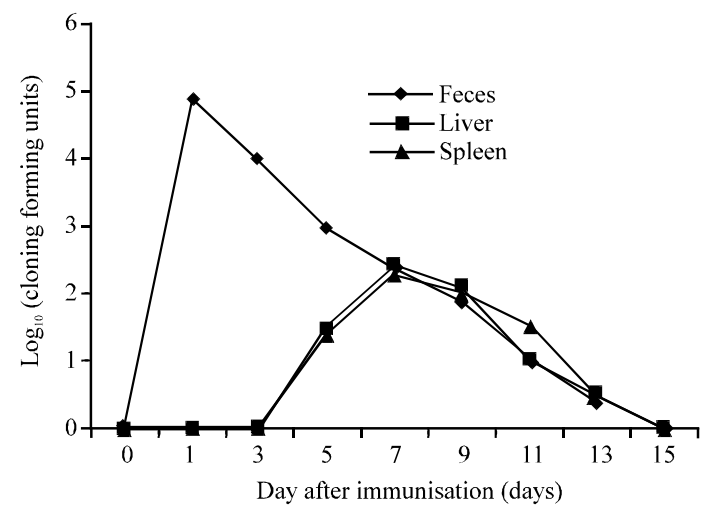

Fig. 6: In vivo stability of recombinant plasmids in mice. This group of mice were orally immunised with SC. 500 (pIRES-M-N). 1, 3, 5, 7, 9, 11, 13 and 15 days later, the mice were killed and the numbers of bacteria present in the spleens, livers and feces were determined by viable count. The number of plasmid-bearing bacteria were quantified on LB agar containing antibiotic. Each point represents the value of logarithm of the total count of plasmid-bearing bacteria in the group of immunised mice

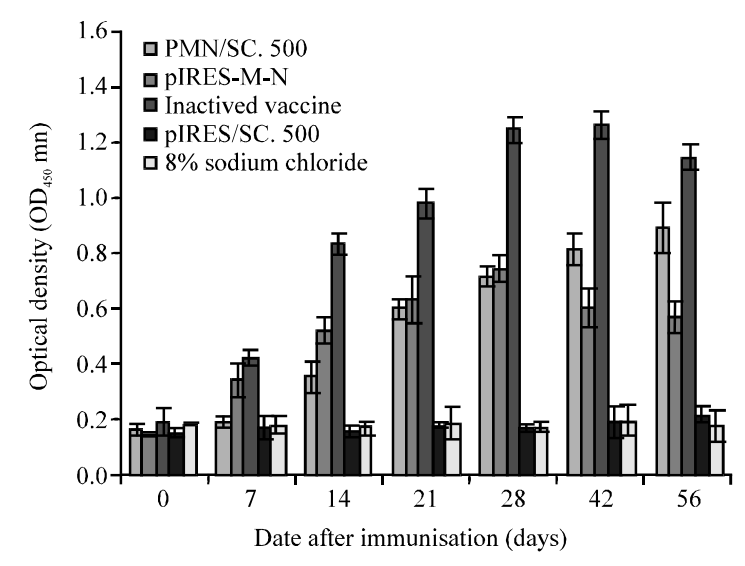

Fig. 7: Levels of specific anti-PEDV IgG responses to all immunized group as measured by ELISA. Values shown for each group are the mean $\pm \mathrm{SE}$ of antibody levels $(n=5)$

stimulate the mice to generate PEDV-specific IgG. None of the mice immunized with SC. 500 (pIRES) or sodium chloride showed significantly detectable specific responses.

IL-4 and IFN- $\boldsymbol{\gamma}$ cytokine ELISA: The IL-4 and IFN- $\boldsymbol{\gamma}$ cytokine levels in sera which obtained from above test were measured using PharMingen OptEIA ${ }^{\text {TM }}$ 


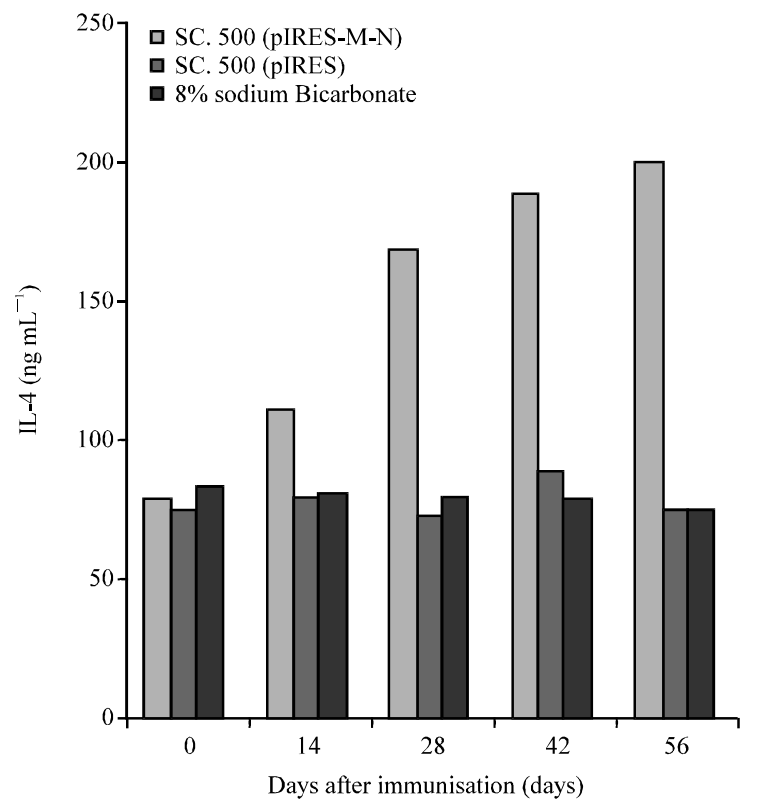

Fig. 8: Level of IL-4 in blood of mice

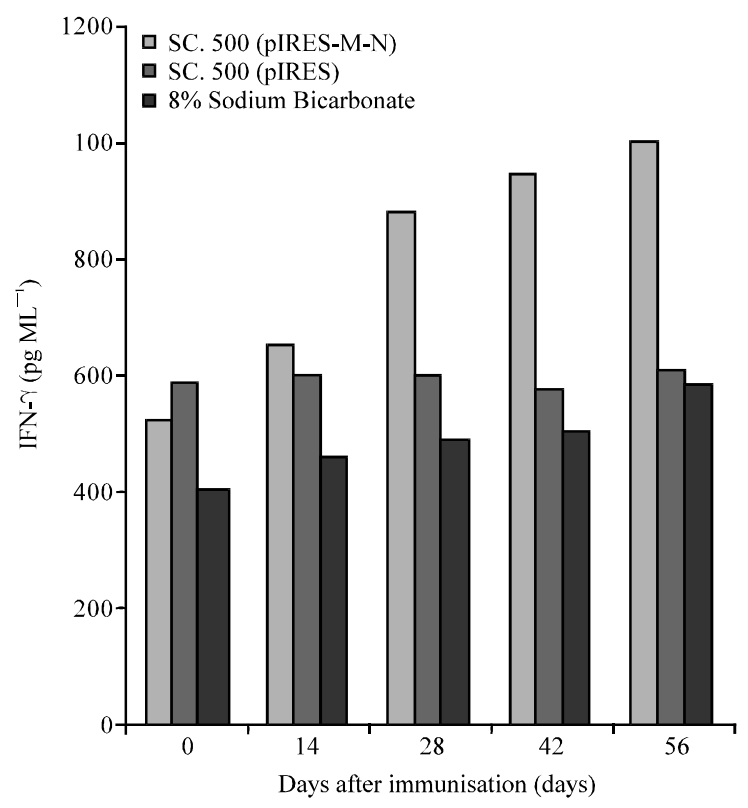

Fig. 9: Level of IFN- $\gamma$ in blood of mice

ELISA kits. IL-4 and IFN- $\gamma$ cytokine levels of all immunized groups were increased obviously while group immunized with SC. 500 (pIRES-M-N) showed much higher level than that immunized with SC. 500 (pIRES) $(\mathrm{p}<0.01)$. Results confirmed that SC. 500 (pIRES-M-N) can significantly enhance the ability of splenic lymphocyte in peripheral blood to induce IL-4 and IFN- $\gamma$ (Fig. 8 and 9).

\section{DISCUSSION}

From its founding until the present day, DNA vaccines have been used to persistently express recombinant protein in vivo and induce significant humoral and cellular immunity (Cardoso et al., 1996). It has become increasingly interesting as a way to prevent infections with many kinds of pathogens. Traditional naked DNA vaccination is usually ineffective, requiring multiple administrations and high dosages of DNA (Yang et al., 2010). As a antigen carrier, the attenuated salmonella delivered DNA vaccine enters the epithelial lining of the gut by invading intestinal $\mathrm{M}$-cells and infects underlying Antigen Presenting Cells (APC) such as macrophages and dendritic cells acted as a natural adjuvant by the presence of pathogen-associated molecular patterns (Monack et al., 2004; Cazorla et al., 2008).

The attenuated bacteria also bring the target DNA to internal organs and lymph tissues (Niess et al., 2005). This new kind of genetic engineering vaccine is so safe and effective and has been demonstrated by a lot of researches for recent years. James E. Galan's experiment support the feasibility of a modified preexposure vaccination strategy wherein a well tolerated $S$. typhi live vector anthrax vaccine is administered to the high-risk population to prime them immunologically to mount rapid, protective serum TNA responses within a few days of receiving a single dose of PA-based vaccine (Galen et al., 2009). In another study, an attenuated Salmonella enterica serovar Typhimurium strain was used as a carrier for Open Reading Frame 7 (ORF7) protein of Porcine Reproductive and Respiratory Syndrome Virus (PRRSV) induced the persistently effctive systemic and mucosal responses in mice (Han et al., 2011). However, this strategy of bacterially delivered immunization to PEDV had rarely been documented in the literature. Therefore, in this study, researchers constructed a plasmid, designated pIRES-M-N, containing two main structural protein genes, nucleocapsid protein gene and membrane protein gene of PEDV. The result of immuno fluorescence and western bloting suggested that two structural protein gene successfully expressed in Vero cells. Then, the recombinant plasmid have been electroporated into $S$. choleraesuis $\mathrm{C} 500$ and detected the stability in vitro. At lastr orally immunized experimental mice with recombinant attenuated Salmonella and detected the index of immunology by ELISA.

Detection of anti-PEDV IgG in sera from SC. 500 (pIRES-M-N) immunized mice indicates that recombinant 
plasmid likely reached appropriate lymphoid tissues to stimulate a systemic immune response. There was however, a difference in PEDV-spicific IgG immune responses stimulated by naked DNA pIRES-M-N or SC. 500 (pIRES-M-N). In the end point titer analyses at the 5 weeks time point, the anti-PEDV IgG titer induced by SC. 500 (pIRES-M-N) was much higher than thatinduced by naked DNA pIRES-M-N, suggesting that SC. 500 (pIRES-M-N) can persistently stimulate the mice to generate PEDV-specific IgG by antigen submission function of the Attenuated Salmonella which naked DNA can not do.

In addition, IL-4 and IFN- $\gamma$ cytokine levels of SC. 500 (pIRES-M-N) immunized mice in sera detected by ELISA. The result showed that both the levels of Thl cytokines IFN- $\gamma$ and Th2 cytokines IL- 4 were elevated in sera of immunized mice, suggesting that SC. 500 (pIRES$\mathrm{M}-\mathrm{N}$ ) could induce both humoral and cellular immunity in mice. Because of IL-4 can enhance CD8 T cell expansion during an immune response (Acacia de Sa Pinheiro et al., 2007) and FN- $\gamma$ can reciprocally to IL-4 counteracting an IL-4 induced reduction in IFN- $\gamma$ production (Apte et al., 2010), it also implied that the recombinant bacteria induced a synthetical immune response in mice.

\section{CONCLUSION}

To the knowledge, this is the first report that Salmonella-delivered $P E D V M$ and $N$ gene as a antigen can induces a persistently synthetical immune response in mice. It provides reference for following study direction on PEDV vaccine.

\section{ACKNOWLEDGEMENT}

This study was supported by Program for Changjiang Scholars and Innovative Research Team in University with grant on IRTO848.

\section{REFERENCES}

Acacia de Sa Pinheiro, A., A. Morrot, S. Chakravarty, M. Overstreet, J.H. Bream, P.M. Lrusta and F. Zavala, 2007. IL-4 induces a wide-spectrum intracellular signaling cascade in CD8+T cells. J. Leukocyte Biol., 81: 1102-1110.

Apte, S.H., P. Groves, S. Olver, A. Baz, D.L. Doolan, A. Kelso and N. Kienzle, 2010. IFN- $\gamma$ inhibits IL-4induced type 2 cytokine expression by CD $8 \mathrm{~T}$ cells $\mathrm{In}$ vivo and modulates the anti-tumor response. $\mathrm{J}$. Immunol., 185: 998-1004.
Cardoso, A.I., M. Blixenkrone-Moller, J. Fayolle, M. Liu, R. Buckland and T.F. Wild, 1996. Immunization with plasmid DNA encoding for the measles virus hemagglutinin and nucleoprotein leads to humoral and cell-mediated immunity. Virology, 225: 293-299.

Cazorla, S.I., P.D. Becker, F.M. Frank, T. Ebensen and M.J. Sartori et al., 2008. Oral vaccination with Salmonella enteric as a cruzipain-dna delivery system confers protective immunity against Trypanosoma cruzi. Infection Immunity, 76: 324-333.

Chen, J.F., D.B. Sun, C.B. Wang, H.Y. Shi and X.C. Cui et al., 2008. Molecular characterization and phylogenetic analysis of membrane protein genes of porcine epidemic diarrhea virus isolates in China. Virus Genes, 36: 355-364.

Chen, X.Y., M.D. John, L.L. Jiang, J.B. Shuai and W.H. Fang, 2005. Stability of recombinant plasmids in attenuated Salmonella typhimurium and their effects on bacterial invasiveness and multiplicaition. Acta Microbiol. Sin., 45: 744-747.

Dietrich, G., S. Spreng, I. Gentschev and W. Goebel, 2009. Bacterial systems for the delivery of eukaryotic antigen expression vectors. Antisense Nucleic Acid Drug Dev., 10: 391-399.

Dunstan, S.J., C.P. Simmons and R.A. Strugnell, 2003. In vitro and In vivo stability of recombinant plasmids in a vaccine strain of Salmonella enteric var. Typhimurium. FEMS Immunol. Med. Microbiol., 37: 111-119.

Galen, J.E., M. Chinchilla, M.F. Pasetti, J.Y. Wang and L. Zhao et al., 2009. Mucosal immunization with attenuated Salmonella enteric serovar Typhi expressing protective antigen of anthrax toxin (PA83) primes monkeys for accelerated serum antibody responses to parenteral PA83 vaccine. J. Infectious Dis., 199: $326-335$.

Ge, J., Y. Jiang, M. Wang, X. Qiao, M. Liu, L. Tang and $\mathrm{Y}$. Li, 2009. Induction of immune response after oral inoculation of mice with Lactobacillus casei surfacedisplayed porcine epidemic diarrhea viral $\mathrm{N}$ protein. Chin. J. Biotechnol., 25: 813-818.

Han, Y.W., S.B. Kim, M. Rahman, E. Uyangaa and B.M. Lee et al., 2011. Systemic and mucosal immunity induced by attenuated Salmonella enteric serovar Typhimurium expressing ORF7 of porcine reproductive and respiratory syndrome virus. Comp. Immunol. Microbiol. Infectious Dis., 34: 335-345.

Jinghui, F. and L. Yijing, 2005. Cloning and sequence analysis of the $\mathrm{m}$ gene of porcine epidemic diarrhea virus LJB/03. Virus Genes, 30: 69-73. 
Kang, T.J., J.E. Seo, D.H. Kim, T.G. Kim, Y.S. Jang and M.S. Yang, 2005. Cloning and sequence analysis of the Korean strain of spike gene of porcine epidemic diarrhea virus and expression of its neutralizing epitope in plants. Protein Expr. Puif., 41: 378-383.

Kweon, C.H., B.J. Kwon, J.G. Lee, G.O. Kwon and Y.B. Kang, 1999. Derivation of attenuated Porcine Epidemic Diarrhea Virus (PEDV) as vaccine candidate. Vaccine, 17: 2546-2553.

Lee, H.K. and S.G. Yeo, 2003. Cloning and sequence analysis of the nucleocapsid gene of porcine epidemic diarrhea virus Chinju99. Virus Genes, 26: 207-212.

Monack, D.M., A. Mueller and S. Falkow, 2004. Persistent bacterial infections: The interface of the pathogen and the host immune system. Nat. Rev. Microbiol., 2: 747-765.

Niess, J.H., S. Brand, X. Gu, L. Landsman and S. Jung et al, 2005. CX3CR1-mediated dendritic cell access to the intestinal lumen and bacterial clearance. Science, 307: 254-258.

Sato, T., N. Takeyama, A. Katsumata, K. Tuchiya, T. Kodama and K.I. Kusanagi, 2011. Mutations in the spike gene of porcine epidemic diarrhea virus associated with growth adaptation in vitro and attenuation of virulence in vivo. Virus Genes, 43: $72-78$.
Schodel, F., 1990. Oral vaccination using recombinant bacteria. Semin. Immunol., 2: 341-349.

Sergeev, O.V., T.I. Aliper, A.N. Mukhin and V.A. Sergeev, 2010. Trials of an attenuated strain of Porcine Epizootic Diarrhea Virus (PEDV) under experimental conditions. Vet. Med. J., 1: 21-25.

Sirard, J.C., F. Niedergang and J.P. Kraehenbuhl, 1999. Live attenuated Salmonella: A paradigm of mucosal vaccines. Immunol. Rev., 171: 5-26.

Sun, D.B., L. Feng, H.Y. Shi and J.F. Chen, 2006. Progress on molecular biology of porcine epidemic diarrhea virus. Prog. Vet. Med., 27: 11-14.

Yang, Y., Z. Zhang, J. Yang, X. Chen, S. Cui and X. Zhu, 2010. Oral vaccination with Ts87 DNA vaccine delivered by attenuated Salmonella typhimurium elicits a protective immune response against Trichinella spiralis larval challenge. Vaccine, 28: 2735-2742.

Zhang, Z.B., J.F. Chen, H.Y. Shi, X.J. Chen and L. Feng, 2011. Expression of the whole $M$ protein of porcine epidemic diarrhea virus $\mathrm{CH} / \mathrm{S}$ strain in Escherichia coliand analysis of its reactogenicity. Chin. Vet. Sci., 41: 31-35. 